\title{
RE2C: A More Versatile Scanner Generator
}

\author{
PETER BUMBULIS and DONALD D. COWAN \\ University of Waterloo
}

\begin{abstract}
It is usually claimed that lexical analysis routines are still coded by hand, despite the widespread availability of scanner generators, for efficiency reasons. While efficiency is a consideration, there exist freely available scanner generators such as GLA [Gray 1988] that can generate scanners that are faster than most hand-coded ones. However, most generated scanners are tailored for a particular environment, and retargeting these scanners to other environments, if possible, is usually complex enough to make a hand-coded scanner more appealing. In this paper we describe $\mathrm{RE} 2 \mathrm{C}$, a scanner generator that not only generates scanners that are faster (and usually smaller) than those produced by any other scanner generator known to the authors, including GLA, but that also adapt easily to any environment.
\end{abstract}

Categories and Subject Descriptors: D.3.2 [Programming Languages]: Language Classifications-specialized application languages; D.3.4 [Programming Languages]: Processors

General Terms: Algorithms, Languages, Performance

Additional Key Words and Phrases: Lexical analysis, scanner generator

\section{INTRODUCTION}

Lexical analysis routines are still often coded by hand despite the widespread availability of scanner generators. For example, while most UNIX ${ }^{\mathrm{TM}}$ systems have a scanner generator installed (typically LEX [Lesk 1975] or flex [Paxson 1988]), few UNIX applications use a mechanically generated scanner. One commonly cited reason for not using LEX-generated scanners is performance: They can be 10 times slower than equivalent hand-coded scanners [Jacobson 1987]. As a result, there has been considerable research into improving the performance of mechanically generated scanners [Paxson 1988; Gray 1988; Grosch 1989]. GLA [Gray 1988], one such scanner generator, can produce scanners that are faster than most hand-coded scanners. However, the use of hand-coded scanners is still prevalent. One possibility is that this is due to the difficulty of adapting the generated scanners to specific applications.

Most scanner generators are tailored to a particular environment. In fact, the trend in recent years has been to integrate scanner generators with compiler toolkits. For example, GLA is part of the Eli compiler construction system [Gray et al. 1992], and Rex [Grosch 1989] is part of the GMD Toolbox

Authors' address: Computer Science Department and Computer Systems Group, University of Waterloo, Waterloo, Ontario, Canada N2L 3G1.

Permission to copy without fee all or part of this material is granted provided that the copies are not made or distributed for direct commercial advantage, the ACM copyright notice and the title of the publication and its date appear, and notice is given that copying is by permission of the Association for Computing Machinery. To copy otherwise, or to republish, requires a fee and /or specific permission.

(c) 1993 ACM 1057-4514/93/0300-0070\$03.50

TM UNIX is a registered trademark of AT \& T Bell Laboratories.

ACM Letters on Programming Languages and Systems,

Vol. 2, Nos. 1-4, March-December 1993, Pages 70-84. 
for Compiler Construction. ${ }^{1}$ Scanners generated by these tools assume the existence of a library of support modules for error handling, input buffering, symbol table management, and similar functions. Although these support modules simplify the task of implementing a compiler or interpreter, they make adaptation to other purposes more difficult. Adaptation to other environments is also made more difficult because, often, assumptions are made about the input and restrictions are placed on tokens in order to achieve better performance. RE2C goes to the other extreme: it concentrates solely on generating code for matching regular expressions.

RE2C is successful at its task: not only does it produce scanners that are faster than those created by other scanner generators, but surprisingly, they are usually smaller as well. Furthermore, RE2C does not make any assumptions about the input or place any restrictions on tokens. To a large degree, the performance and flexibility of RE2C-generated scanners are due to a novel method for determining when to refill a buffer that avoids the complications introduced by the sentinel method [Aho et al. 1988].

The following sections describe RE2C scanner specifications, discuss how these specifications are converted into scanners, and give performance results achieved by our implementation (including a comparison with GLA).

\section{SCANNER SPECIFICATIONS}

An RE2C source file consists of C [Kernighan and Ritchie 1988] or C ++ [Ellis and Stroustrup 1990] ${ }^{2}$ code interleaved with comments of the form $/ *$ !re2c...* / containing scanner specifications. These specifications are replaced with generated code that is invoked simply by "falling into" the comments, as illustrated in Figure 1 and in Appendix A. ${ }^{3}$

A scanner specification takes the form of a list of rules, each rule consisting of a regular expression [Harrison 1978] and an action expressed in executable code. Figure 2 illustrates a trivial RE2C scanner specification that is used as an example throughout this paper. Each call to the code generated from a specification will first determine the longest possible prefix of the remaining input that matches one of the regular expressions and will then execute the action in the first applicable rule.

RE2C is different from most other scanner generators in that the user must provide the input buffering mechanism for the scanner; the generated code simply assumes that the user has defined three pointers: YYCURSOR, YYLIMIT, and YYMARKER, and a routine YYFILL $(n)$. Before executing the generated code, YYCURSOR and YYLIMIT must be set to point to the first and one past the last character in the buffer, respectively. After a token is recognized and before any action is executed, YYCURSOR is set to point to just past the token. YYFILL will be called as the buffer needs filling; at least $n$ additional input characters should be provided. When YYFILL is called, YYCURSOR will point to the next character to be scanned, and YY-

\footnotetext{
${ }^{1}$ Also known as Cocktail (Compiler-Compiler-Toolbox Karlsruhe).

${ }^{2}$ Retargeting RE2C to a different language is straightforward.

${ }^{3} \mathrm{RE} 2 \mathrm{C}$-generated scanners require no additional support code.
} 


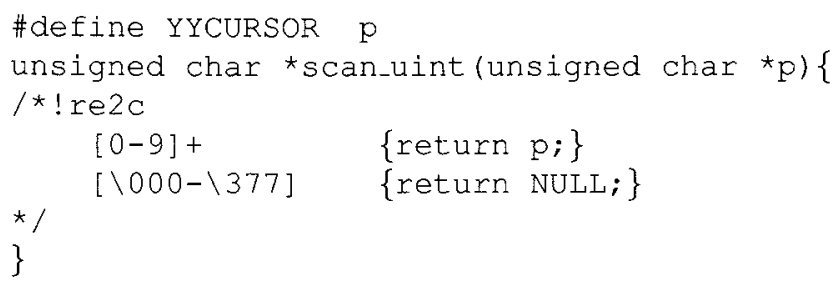

Fig. 1. Simple scanner.

$\begin{array}{lll}\text { print" } & \{\text { return DRINT; } & / \text { rule } 5 * /\} \\ {[a-z]+} & \{\text { return ID; } & / \star \text { rule } 4 * /\} \\ {[0-9]+} & \{\text { return DEC; } & / \star \text { rule } 3 * /\} \\ \text { "0x" [0-9a-f]+ } & \{\text { return HEX; } & \text { * rule } 2 \star /\} \\ {[\backslash 000-\backslash 377]} & \{\text { return ERR; } & / \star \text { rule } 1 * /\}\end{array}$

Fig. 2. Sample specification: $[a-b]$ matches any character between $a$ and $b$, inclusively. The last rule, for example, will match any eight-bit character. Rules are listed in order of precedence.

MARKER, if set, will point to a possible backtracking point in the buffer. YYFILL must update YYLIMIT, and possibly YYCURSOR and YYMARKER before returning. Typically YYCURSOR, YYLIMIT, YYMARKER, and YYFILL $(n)$ will be defined as macros.

\subsection{Things that RE2C Does Not Provide}

$\mathrm{RE} 2 \mathrm{C}$ does not provide many things available in more conventional scanner generators, including default rules, end-of-input pseudotokens, and buffer management routines. All of these must be supplied by the user. Rather than being a handicap, this allows RE2C-generated scanners to be tailored to almost any environment. For example, the scanner defined in Figure 1 compiles into 32 bytes of $i 486$ code (using Watcom C 9.5), the same size as an equivalent hand-coded routine. Most other scanner generators cannot produce scanners that are competitive with hand-coded analyzers in this case. Furthermore, it is not overly difficult to implement a more traditional scanner using RE2C. For example, Appendix A contains the support code for the $\mathrm{C}$ scanner benchmarked in Table I (shown later). Note that this code allows for arbitrarily long contiguous tokens and provides line- and column-number information.

\section{GENERATING DIRECTLY EXECUTABLE SCANNERS}

As demonstrated by GLA [Gray 1988], generating directly executable code instead of tables can result in much faster scanners. However, to achieve this speed, GLA-generated scanners make some assumptions about the input and place certain restrictions on tokens. ${ }^{4}$ In this section we show how to generate directly executable scanners that not only avoid such restrictions, but are also faster and usually smaller. The approach presented here has the added benefit that even faster scanners can easily be created, at the expense of increased code size, by using a technique akin to loop unrolling.

\footnotetext{
${ }^{4}$ These assumptions and restrictions are discussed in more detail in Sections 3.3.1 and 5.1. ACM Letters on Programming Languages and Systems, Vol. 2, Nos. 1-4, March-December 1993.
} 


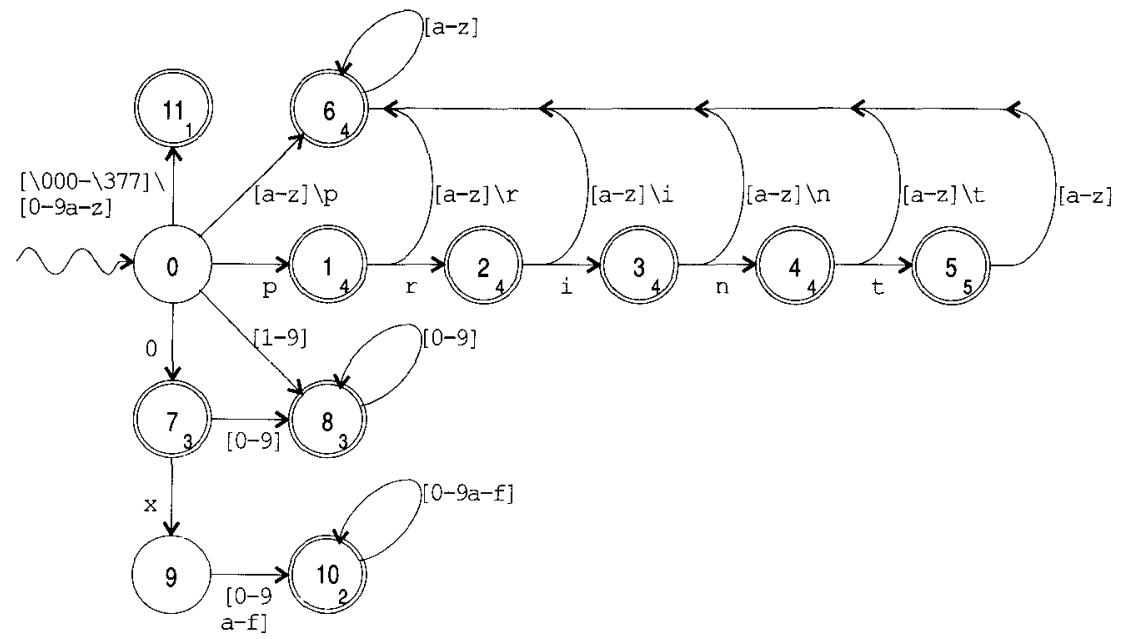

Fig. 3. DFA for sample specification in Figure 2: State 0 is the start state. Accepting states are labeled with the number of the rule that they match. For example, state 10 accepts rule 2 . Transitions differing only by label are represented with the same arc. For example, state 0 has transitions to state 6 on all of the following characters: $\mathbf{a}, \ldots, \mathbf{o}, \mathbf{q}, \ldots, \mathbf{z}$.

\subsection{Constructing a DFA}

The first step in generating a directly executable scanner is to construct a DFA that recognizes the regular expressions in the specification. Figure 3 presents a DFA that recognizes the regular expressions in Figure 2. One possible algorithm for constructing such a DFA can be found in Aho et al. [1988]. Given such a DFA, the task of scanning the input can be expressed as follows:

- Starting from the start state, move from state to state along transitions labeled with consecutive characters from the input. When no further transitions can be made, backtrack to the last accepting state, say, $q$. The path to $q$ spells the next token, and the rule associated with $q$ determines the code to be executed.

As a result, the problem of generating scanners essentially reduces to the problem of generating an executable representation for a DFA.

\subsection{Generating Code}

If we assume that the input is entirely contained in a single buffer, then generating code for the DFA is relatively straightforward, as illustrated by the code templates in Figure 4. Note that the only difference between the templates for accepting and nonaccepting states is that the accepting states have additional code to save backtracking information. Figure 5 shows code that might be generated for state 1 in Figure 3.

\subsection{Buffering}

Complications arise when the input is not contained in a single buffer: additional code is needed for filling the buffer. 


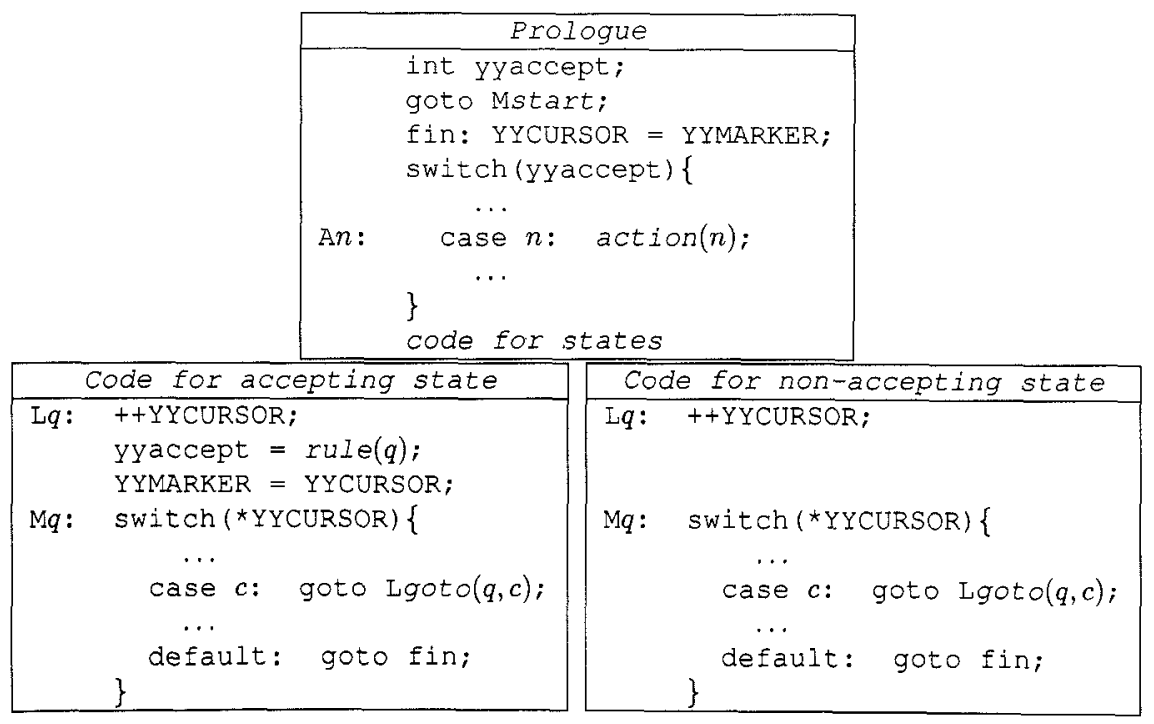

Fig. 4. Directly executable scanner: The code generated for a scanner consists of a prologue followed by code for each state. start is the start state, action $(n)$ denotes the code associated with rule $n, \operatorname{got} o(q, c)$ denotes the state reached from state $q$ along the transition labeled with $c$, and rule $(q)$ denotes the rule associated with state $q$. yyaccept is used to save backtracking information. The M-labels will be used in Section 3.4.2.

Fig. 5. Code for state 1.

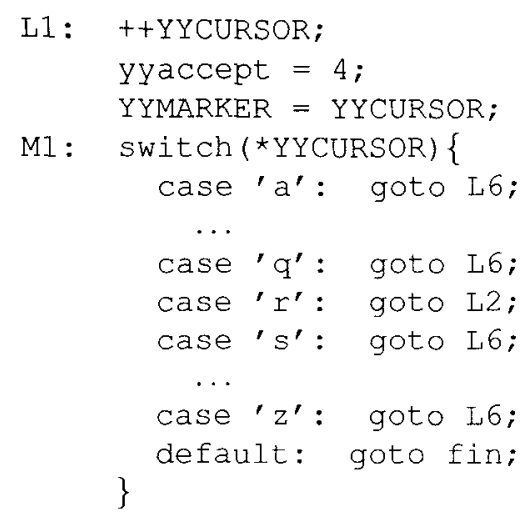

3.3.1 The Sentinel Method. Most scanner generators use the sentinel method [Aho et al. 1988] to determine when the buffer needs filling. In the simplest case, a symbol that does not appear in valid input is chosen as the sentinel character. An extra state is added to the DFA, and transitions to this state on the sentinel symbol are added to the original states. When the DFA arrives in this new state, it is time to refill the buffer. After the buffer is refilled, scanning must be restarted in the previous state. Unfortunately, this is not possible when the approach outlined in Figure 4: the necessary information is simply not available. Code could be added to each state to save the necessary information, but this would result in slower and larger scan- 


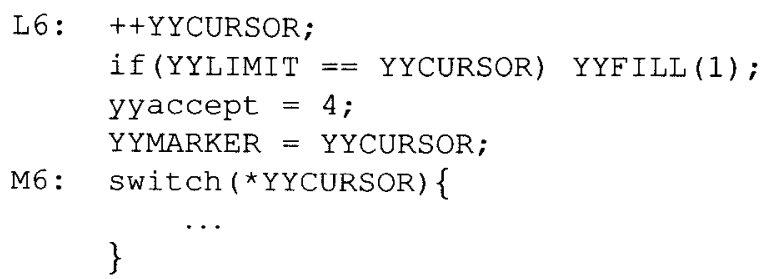

Fig. 6. Code for state 6.

ners. GLA solves this problem by ensuring that the ${ }^{-}$sentinel only gets inserted between tokens: if this is the case, the scanner can always be restarted from the start state. To ensure that the sentinel only gets inserted between tokens, GLA allows new-line (ASCII LF) characters to appear only at the end of a token and disallows the buffering of partial lines. ${ }^{5}$

3.3.2 Buffering. RE2C-generated scanners check if the buffer needs filling simply by comparing YYCURSOR and YYLIMIT. A method inspired by the mechanism used to guard against stack overflow in Pennello [1986] ${ }^{6}$ is used to reduce the amount of checking.

Checks are only inserted in certain key states. These checks simply ensure that there is enough input in the buffer for the scan to proceed until the next key state. For example, in the DFA of Figure 3 it is sufficient to check that there are at least six characters in the buffer when it starts, and that there is at least one character in the buffer when the DFA is in states 6,8 , or 10 . No other checks are required. The checks inserted in key states are of the form

if((YYLIMIT - YYCURSOR) $<n)$ YYFILL $(n)$;

where $n$ is the maximum number of characters that can be consumed before another key state is reached. For example, Figure 6 shows the code generated for state 6 in Figure 3.

A set of key states can be determined by discovering the strongly connected components (SCCs) of the DFA. An SCC is a maximal subset of states such that there exists a path from any state in the subset to any other. The set of key states consists of all of the states in nontrivial SCCs, together with the start state. Note that for each SCC $S$ we actually only have to include a subset of states of $S$ such that when the subset is removed $S$ becomes acyclic. Indeed, Pennello [1986] described a simple heuristic for choosing such a subset. However, since in practice most of the (nontrivial) SCCs encountered will consist of a single state, the current version of RE2C simply includes all states in nontrivial SCCs. ${ }^{7}$ An algorithm given by DeRemer and Pennello [1982] was used to compute the SCCs.

\footnotetext{
${ }^{5}$ If the input contains no new-lines, a GLA scanner will attempt to buffer the entire input stream.

${ }^{6}$ The problem of detecting stack overflow in LR parsers is probably best left to hardware mechanisms [Horspool and Whitney 1990].

${ }^{7}$ It should be noted that finding the minimal set of states to remove from an SCC in order to render it acyclic is equivalent to the feedback vertex set problem, which is NP-complete [Garey and Johnson 1991].
} 
Fig. 7. Code for state 1 with backtracking elimi-

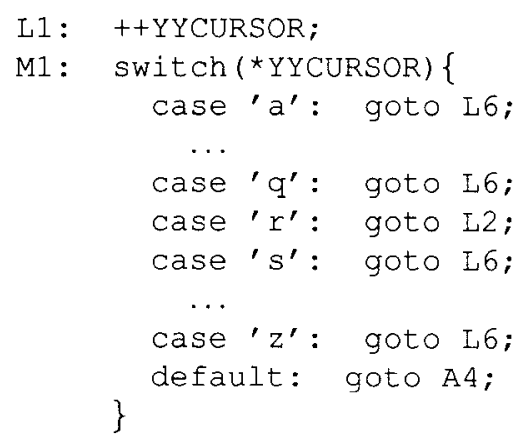

\subsection{Optimizations}

Even good optimizing $\mathrm{C}$ compilers can be coaxed into generating much smaller and slightly faster code if some transformations are first applied to the generated code.

3.4.1 Eliminating Backtracking. Consider state 1 in the DFA in Figure 3. Note that, since all of the transitions from state 1 reach only accepting states, backtracking information does not need to be saved if the code for the default case is changed to go directly to the code associated with state 1 . The result of this optimization is shown in Figure 7. More generally, this optimization can be applied to all accepting states that have transitions only to accepting states.

3.4.2 Optimizing switches. Most $\mathrm{C}$ compilers generate either a jump table or a set of if statements for a switch statement, depending on the distribution of the case labels. In many compilers the decision as to which method to use is biased toward generating jump tables, since in most cases this results in faster, albeit larger, code. However, experience with directly executable scanners has shown that replacing many of these jump tables with if statements results in scanners that are much smaller and, surprisingly, in some cases slightly faster as well. ${ }^{8}$ As a result, the capability of replacing a switch statement with if statements was added to RE2C.

RE2C bases its decision of whether to generate a switch statement or to replace it with ifs solely on the density ${ }^{9}$ of the switch statement. It is surprising that such a simple heuristic works well. For more esoteric applications in which the input alphabet is not a simple interval, RE2C has the advantage in that there is no provision for "don't care" entries in a switch statement: If no case matches, none of the statements in the switch must be executed. However, for the examples in Table I this is not so: RE2C simply does a better job of generating code for switch statements than the compiler. Sale [1981], Hennessy and Mendelsohn [1982], and Bernstein [1985] also addressed the problem of generating good code for switch statements.

\footnotetext{
${ }^{8}$ See Table I for examples.

${ }^{9}$ The number of distinct subranges divided by the total number of cases. 


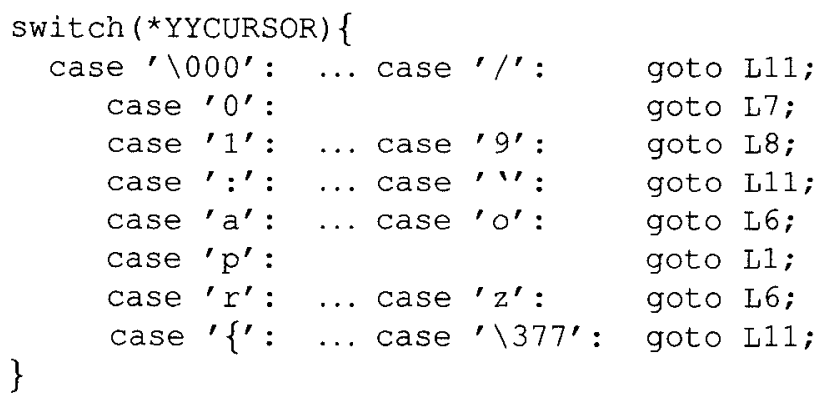

Fig. 8. switch for state 0 .

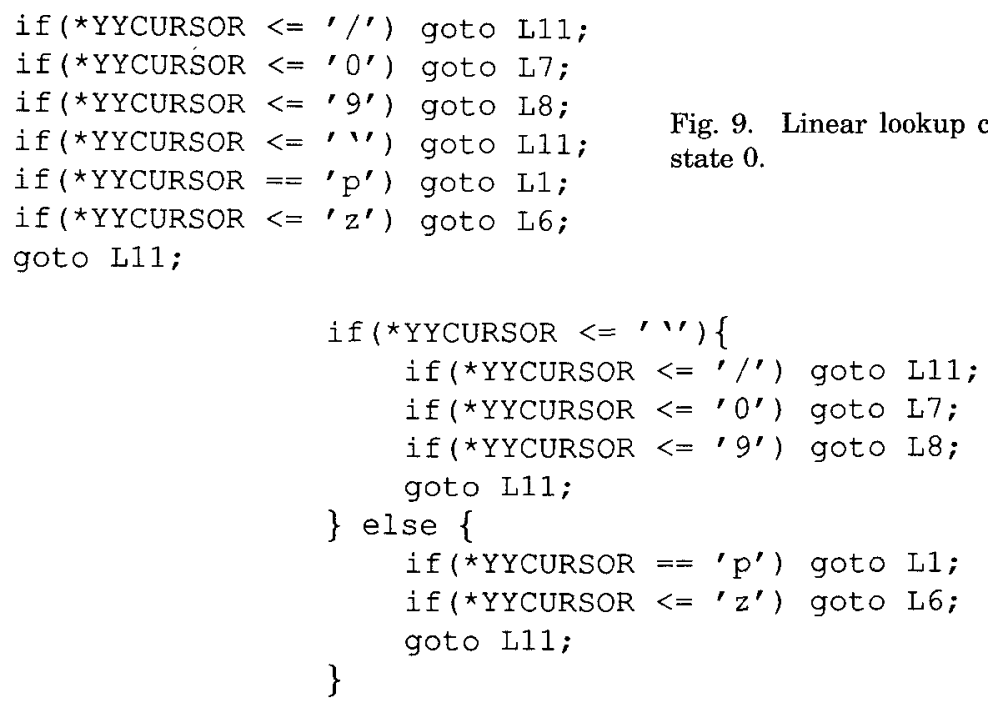
state 0 .

Fig. 9. Linear lookup code sequence for

Fig. 10. Binary lookup code sequence for state 0 .

Replacing switches with ifs. When replacing a switch statement with if statements, it is useful to sort the cases by label and then to group them according to rule into subranges, as illustrated in Figure 8. RE2C replaces a switch with either a linear or binary search, depending on the number of subranges in the switch. If there are only a few subranges, a linear search is generated; otherwise, a binary search is used.

Figures 9 and 10 show linear and binary searches, respectively, that could be used to replace the switch in Figure 8. Note, in particular, the comparison for the $\mathbf{p}$ in Figure 9. This optimization eliminates a comparison each time it is applied. Also note that no comparisons are required at the top and bottom of the range.

Simplifying switches. As a general rule, better replacement code can be generated for a switch if it contains fewer subranges. One way of reducing the number of subranges in a switch, at the expense of some speed, is to locate a base switch that is very similar and then to replace the code for all cases that appear identically in the base switch with a goto to (the code 
Fig. 11. Code for states 1-4 after all optimizations.

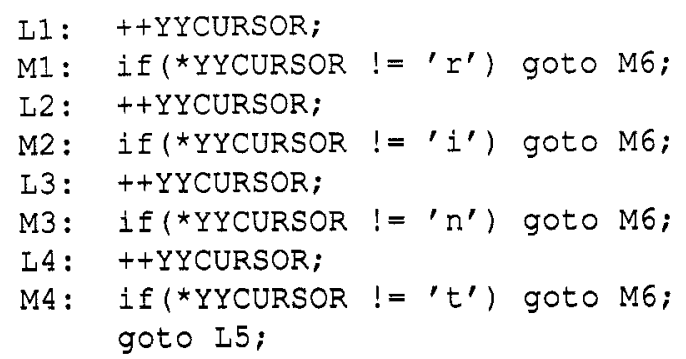

generated for) the base switch. RE2C uses this optimization to good advantage when generating code in the transitions of states used for matching keywords. For example, note that the switches for states 1-4 differ from the switch of state 6 only on $\mathbf{r}, \mathbf{i}, \mathbf{n}$, and $\mathbf{t}$, respectively. Figure 11 shows the code generated for these states. Another way of implementing this optimization is to construct a tunnel automaton [Grosch 1989] from the DFA and then to generate code from the tunnel automaton.

Common Subexpression Elimination. Many compilers will miss the fact that $*$ YYCURSOR in Figures 9 and 10 should be loaded into a register. Most can be coaxed to do so by first assigning $*$ YYCURSOR to a local variable.

\section{EXPERIMENTAL RESULTS}

Table I compares two RE2C-generated C scanners with the (hand-coded) lcc scanner [Fraser and Hanson 1991] and comparable GLA- and flex-generated scanners on a variety of platforms. It reports the times in seconds required by the various scanners to scan about 170,000 lines of $\mathrm{C}$ source. The $5,607,820$ byte source file used essentially consists of 10 copies of the source to James Clark's SGML parser, sgmls. ${ }^{10}$ The times reported are averages for 10 trials; the sizes reported include everything but C library code ${ }^{11}$ flex provides a number of table-compression options, including - Cem for tables optimized for space and $-\mathbf{C f}$ for tables optimized for speed. By default, RE2C will use a heuristic to decide if a switch should be replaced with ifs: The $-\mathbf{s}$ option forces RE2C to generate switches always.

To make comparisons more meaningful, all semantic processing code was removed from the GLA-generated and lcc scanners, and code to provide lineand column-number information was added to the RE2C specification. The remaining differences of note between the scanners are that

- the flex-generated scanners do not provide line- or column-number information, and

- the GLA-generated scanner assumes 7-bit input.

As a general rule, the RE2C-generated scanners were the fastest, followed by the GLA-generated scanner and then the lcc scanner. The flex-generated

${ }^{10}$ Available for anonymous ftp from ftp.uu.net as /pub/text-processing / sgml / sgmls1.1.tar.Z.

${ }^{11}$ The GLA-generated scanner sizes also do not include the size of an error-reporting module err.o. 
Table I. Comparison of Generated C Scanners

\begin{tabular}{|c|c|c|c|c|c|c|c|}
\hline \multirow[b]{2}{*}{ program } & \multirow{2}{*}{\multicolumn{2}{|c|}{$\begin{array}{l}\text { time } \\
\text { sys }\end{array}$}} & \multicolumn{5}{|c|}{ space } \\
\hline & & & total & text & data & bss & total \\
\hline \multicolumn{8}{|c|}{$R 4000 / g c c 2.3 .3-O$} \\
\hline flex -Cem & 10.36 & 0.87 & 11.23 & 5200 & 4192 & 48 & 9440 \\
\hline flex -Cf & 5.44 & 0.72 & 6.16 & 4688 & 64384 & 48 & 69120 \\
\hline lcc & 3.19 & 0.67 & 3.86 & 7328 & 1216 & 8256 & 16800 \\
\hline gla & 2.89 & 0.63 & 3.52 & 11552 & 3056 & 144 & 14752 \\
\hline re $2 c$ & 2.54 & 0.68 & 3.22 & 13264 & 512 & 0 & 13776 \\
\hline $\mathrm{re} 2 \mathrm{c}-\mathrm{s}$ & 2.38 & 0.67 & 3.05 & 11056 & 4528 & 0 & 15584 \\
\hline \multicolumn{8}{|c|}{$\frac{1}{R 4000 / c c 2.11 .2-0 \text {-Olimit } 5000}$} \\
\hline flex -Cem & 9.97 & 0.89 & 10.86 & $4 \overline{704}$ & 4240 & 32 & 8976 \\
\hline flex -Cf & 6.19 & 0.72 & 6.91 & 4256 & 64432 & 32 & 68720 \\
\hline Icc & 2.74 & 0.72 & 3.46 & 9664 & 864 & 8256 & 18784 \\
\hline gla & 2.46 & 0.69 & 3.15 & 19232 & 2992 & 128 & 22352 \\
\hline re $2 c$ & 2.97 & 0.63 & 3.60 & 15088 & 528 & 0 & 15616 \\
\hline re $2 c-s$ & 2.94 & 0.61 & 3.55 & 16080 & 11808 & 0 & 27888 \\
\hline \multicolumn{8}{|c|}{ SPARC/gcc2.3.3-O } \\
\hline flex-Cem & 16.03 & 2.78 & 18.81 & 8992 & 24 & 48 & 9064 \\
\hline flex -Cf & 7.84 & 2.69 & 10.53 & 6560 & 62232 & 48 & 68840 \\
\hline lcc & 4.46 & 2.01 & 6.47 & 7800 & 384 & 8256 & 16440 \\
\hline gla & 4.08 & 1.56 & 5.64 & 10864 & 2168 & 136 & 13168 \\
\hline $\mathrm{re} 2 \mathrm{c}$ & 3.67 & 1.76 & 5.43 & 13552 & 0 & 0 & 13552 \\
\hline $\mathrm{re} 2 \mathrm{c}-\mathrm{s}$ & 3.48 & 1.70 & 5.18 & 15464 & 0 & 0 & 15464 \\
\hline \multicolumn{8}{|c|}{$i 486 / g c c 2.4 .5-O$} \\
\hline flex-Cem & 21.86 & 1.26 & 23.12 & 8536 & 20 & 24 & 8580 \\
\hline flex $-\mathrm{Cf}$ & 9.12 & 1.18 & 10.30 & 6200 & 62228 & 24 & 68452 \\
\hline lcc & 5.45 & 1.22 & 6.67 & 5924 & 384 & 8240 & 14548 \\
\hline gla & 5.11 & 1.18 & 6.29 & 15496 & 2144 & 108 & 17748 \\
\hline re $2 c$ & 4.73 & 1.13 & 5.86 & 9800 & 0 & 0 & 9800 \\
\hline re $2 c-s$ & 4.85 & 1.17 & 6.02 & 12968 & 0 & 0 & 12968 \\
\hline \multicolumn{8}{|c|}{$68020 / \mathrm{gccl} .40-\mathrm{O}$} \\
\hline flex-Cem & 117.37 & 5.89 & 123.26 & 7700 & 20 & 22 & 7742 \\
\hline flex $-\mathrm{Cf}$ & 50.93 & 5.27 & 56.20 & 5388 & 62228 & 22 & 67638 \\
\hline lcc & 33.28 & 6.28 & 39.56 & 4956 & 384 & 8236 & 13576 \\
\hline gla & 33.80 & 4.20 & 38.00 & 13904 & 2144 & 106 & 16154 \\
\hline re2c & 28.92 & 2.91 & 31.83 & 8556 & 0 & 0 & 8556 \\
\hline $\mathrm{re} 2 \mathrm{c}-\mathrm{s}$ & 30.72 & 3.19 & 33.91 & 9856 & 0 & 0 & 9856 \\
\hline
\end{tabular}

scanners were significantly slower. Only the space-optimized flex scanner was smaller than the default RE2C scanner, and only by a narrow margin. There are some architectures, notably the IBM 370, on which table driven scanners will probably produce better results: IBM 370 compilers typically generate poor code for large routines.

The various scanners and input files used for the tests are available for anonymous ftp from esg.uwaterloo.ca in /pub / peter / re2c / sampler.tar.Z. flex is available for anonymous ftp from ftp.uu.net as /packages / gnu / flex-2.3.7.tar.Z, GLA is available for anonymous ftp from ftp.cs.colorado.edu as part of the Eli package /pub / cs / distribs / eli / Eli3.4.2.tar.Z, and the lcc front end is available for anonymous ftp from princeton.edu as /pub/lcc/lecfe-1.9.tar.Z. An alpha version of RE2C 
will soon be made available for anonymous ftp from csg.uwaterloo.ca as /pub / peter / re2c / re2c-0.5.tar.Z.

\section{RELATED WORK}

The key to the performance and flexibility of an RE2C-generated scanner is the approach used to determine when the buffer needs filling. Interestingly, the lce scanner [Fraser and Hanson 1991] uses a similar approach (with certain concessions to keep the bookkeeping manageable).

\subsection{Comparison with GLA}

It is natural to compare RE2C to GLA [Gray 1988], as it also generates directly executable scanners. RE2C and GLA have many differences simply because they are targeted for different types of users: GLA is intended for people who simply wish to leverage their efforts with existing tools and libraries; RE2C is intended for people who have more specialized needs and are willing to provide their own support routines. For example, GLA provides a good buffering mechanism; RE2C users must supply their own. These differences, however, are not unique to GLA and have been addressed for the most part in previous sections.

Of more interest are the differences in the code that RE2C and GLA generate. Scanners generated by RE2C and GLA differ primarily in two aspects: how they determine when the buffer needs filling, and how they generate code for switches.

GLA uses the ASCII NUL character as the sentinel to determine when the buffer needs filling. To improve the speed and reduce the size of the generated scanners, GLA buffers only complete lines and restricts tokens to those that do not contain new-line (ASCII LF) characters. ${ }^{12}$ If a token with an embedded new-line character (such as a comment) is required, it must be recognized with an auxiliary scanner written in $\mathrm{C}$. This code has to perform the buffering-related bookkeeping that is done automatically by GLAgenerated code.

The mechanism RE2C uses to refill the buffer eliminates these restrictions and yet allows RE2C to generate faster and smaller scanners. RE2C also allows both auxiliary and primary scanners to be specified using regular expressions. For example, Appendix A contains an auxiliary scanner for comments.

Like RE2C, GLA usually replaces switches with ifs. Unlike RE2C, GLA does not use a case-based heuristic to decide which switches to replace: Rather, it always generates a switch for the start state and uses ifs for the rest. GLA replaces switches with code sequences of the following form:

$$
\begin{aligned}
& \text { if }\left(* \text { YYCURSOR in } S_{1}\right) \text { goto } \mathrm{L}_{1} \text {; } \\
& \vdots \\
& \text { if }\left(* \text { YYYURSOR in } S_{n}\right) \text { goto } \mathbf{L}_{n} ;
\end{aligned}
$$

Bit vectors are used for all membership tests involving sets with more than one element. As an optimization, if a state has a transition to itself, the test

\footnotetext{
${ }^{12}$ This is discussed in more detail in Section 3.3.1.

ACM Letters on Programming Languages and Systems, Vol. 2, Nos. 1-4, March-December 1993.
} 


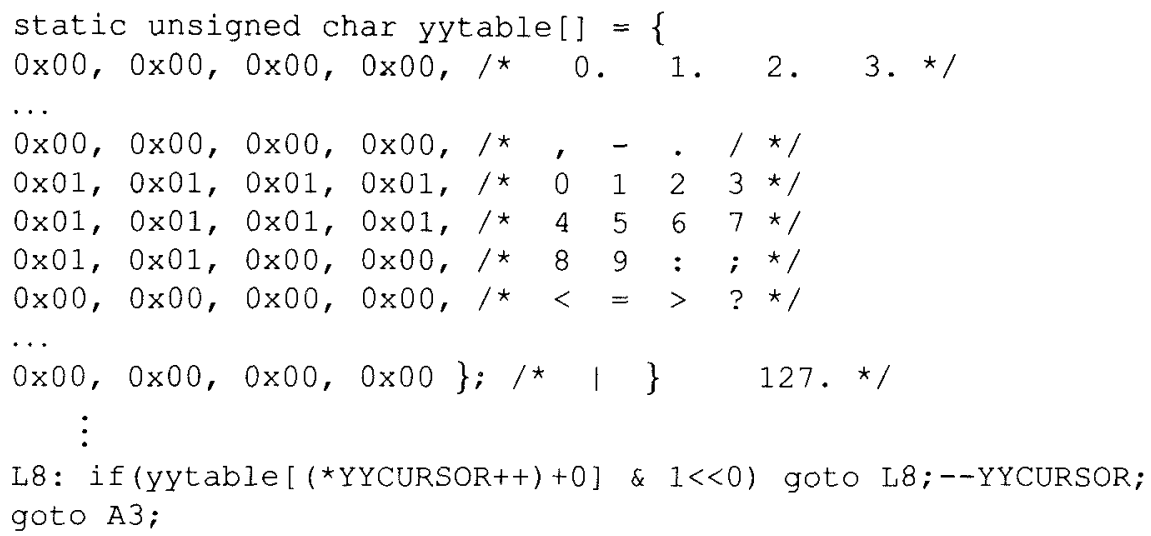

Fig. 12. GLA code for state 8 of Figure 3.

as to whether to remain in the same state or not is performed first. For example, Figure 12 shows the GLA-generated code for state 8 in Figure $3 .^{13}$ Note the use of 128 element entries for the bit vectors to reduce the scanner size: A GLA-generated scanner will crash or otherwise behave unpredictably if a non-ASCII character appears in the source. ${ }^{14}$

In some sense the results of Section 4 are a bit misleading: The GLA specification that was used to obtain the figures in Table I is not a typical GLA specification. Usually, scanners implemented using GLA will handle keywords as identifiers, as GLA has been optimized for this [Gray 1988]. Table II presents a fairer comparison: The keyword-matching rules were removed from both the GLA and RE2C specifications. The RE2C-generated scanners were still faster and smaller except on the MIPS R4000, where the cc-compiled GLA scanner was slightly faster.

Note, however, that the RE2C specification can be substantially sped up by using a technique akin to loop unrolling. Replacing the original keywordmatching rule in the $\mathrm{RE} 2 \mathrm{C}$ specification ${ }^{15}$

with the following rules

\section{LI $* \quad$ \{RET(ID); $\}$}

L

L I

\{RET(ID);

LII

[RET(ID);

LII I

(R

LII I IRET(ID);

L I I I I IRET(ID);

L I I I I I I (RET(ID); ;

L I I I I I I IRET(ID);

$\mathbf{L} \mathbf{I} *$

(RET(ID);)

\footnotetext{
${ }^{13}$ Actually, GLA would generate a while statement. Most compilers will generate the same object code for both.

${ }^{14}$ No checks are made to ensure that only seven-bit characters appear in the input.

${ }^{15} \mathbf{L}=[\mathbf{a}-\mathbf{z A}-\mathbf{Z}-]$ and $\mathbf{I}=[\mathbf{a}-\mathbf{z A}-\mathbf{Z - 0}-\mathbf{9}]$.
}

ACM Letters on Programming Languages and Systems, Vol. 2, Nos. 1-4, March-December 1993. 
Table II. Scanner Performance with Keywords Treated as Identifiers

\begin{tabular}{|c|c|c|c|c|c|c|c|}
\hline \multirow[b]{2}{*}{ program } & \multicolumn{3}{|c|}{ time } & \multicolumn{4}{|c|}{ space } \\
\hline & user & sys & total & text & data & bss & total \\
\hline \multicolumn{8}{|c|}{$R 4000 / \operatorname{gcc} 233-O$} \\
\hline gla & 2.63 & 0.58 & 3.21 & 5040 & 2496 & 144 & 7680 \\
\hline re2c & 2.50 & 0.65 & 3.15 & 6448 & 512 & 0 & 6960 \\
\hline $\mathrm{re} 2 \mathrm{c}-\mathrm{s}$ & 2.49 & 0.67 & 3.16 & 4976 & 4224 & 0 & 9200 \\
\hline $\mathrm{re} 2 \mathrm{c}-\mathrm{s} \dagger$ & 2.08 & 0.59 & 2.67 & 5792 & 4224 & 0 & 10016 \\
\hline \multicolumn{8}{|c|}{$R 4000 /$ cc2.11.2-O-Olımit 5000} \\
\hline gla & $\overline{2.43}$ & 0.64 & 3.07 & 6512 & 2416 & 128 & 9056 \\
\hline re2c & 2.93 & 0.67 & 3.60 & 8048 & 528 & 0 & 8576 \\
\hline $\mathrm{re} 2 \mathrm{c}-\mathrm{s}$ & 3.04 & 0.64 & 3.68 & 9952 & 2208 & 0 & 12160 \\
\hline \multicolumn{8}{|c|}{ SPARC/gcc233-O } \\
\hline gla & 4.08 & 1.65 & 5.73 & 5472 & 1656 & 136 & 7264 \\
\hline $\mathrm{re} 2 \mathrm{c}$ & 3.77 & 1.67 & 5.44 & 7008 & 0 & 0 & 7008 \\
\hline re $2 c-s$ & 3.66 & 2.37 & 6.03 & 9112 & 0 & 0 & 9112 \\
\hline \multicolumn{8}{|c|}{$1486 / \operatorname{gcc} 245-O$} \\
\hline gla & 5.04 & 1.15 & 6.19 & 5368 & 1632 & 108 & 7108 \\
\hline re $2 \mathrm{c}$ & 4.75 & 1.17 & 5.92 & 5448 & 0 & 0 & 5448 \\
\hline re $2 c-s$ & 5.06 & 1.13 & 6.19 & 8248 & 0 & 0 & 8248 \\
\hline \multicolumn{8}{|c|}{$68020 / \mathrm{gccl} .40-O$} \\
\hline gla & 32.69 & 3.37 & 36.06 & 4772 & 1632 & 106 & 6510 \\
\hline re $2 \mathrm{c}$ & 29.86 & 3.74 & 33.60 & 4468 & 0 & 0 & 4468 \\
\hline $\mathrm{re} 2 \mathrm{c}-\mathrm{s}$ & 28.77 & 3.55 & 32.32 & 5616 & 0 & 0 & 5616 \\
\hline
\end{tabular}

${ }^{\dagger}$ Uses an "unrolled" specification.

reduces the number of end-of-buffer checks and results in a significant speed improvement over the GLA-generated scanner.

\section{SUMMARY AND FURTHER WORK}

This paper has described RE2C, a tool for creating lexical analyzers. Unlike other such tools, RE2C concentrates solely on generating efficient code for matching regular expressions. Not only does this singleness of purpose make RE2C more suitable for a wider variety of applications, it allows it to generate scanners that approach hand-crafted scanners in terms of size and speed. Compared to scanners generated by flex and GLA, RE2C-generated scanners are faster and in many cases smaller as well.

Although RE2C-generated scanners perform well, there is still room for improvement. Near-term improvements include using GLA's bit vectors to simplify some switches and adding a state unrolling operator. In the longer term, in-line actions will be added to RE2C. For example, a specification like

$$
D l \mathbf{c}=\$ \mid(D\{\mathbf{c}=\mathbf{1 0} * \mathbf{c}+\$\}) *
$$

might be used to obtain the value of a previously scanned integer. Typically, these sorts of specifications would be used as an action in some other specification. 


\section{APPENDIX A. C SCANNER}

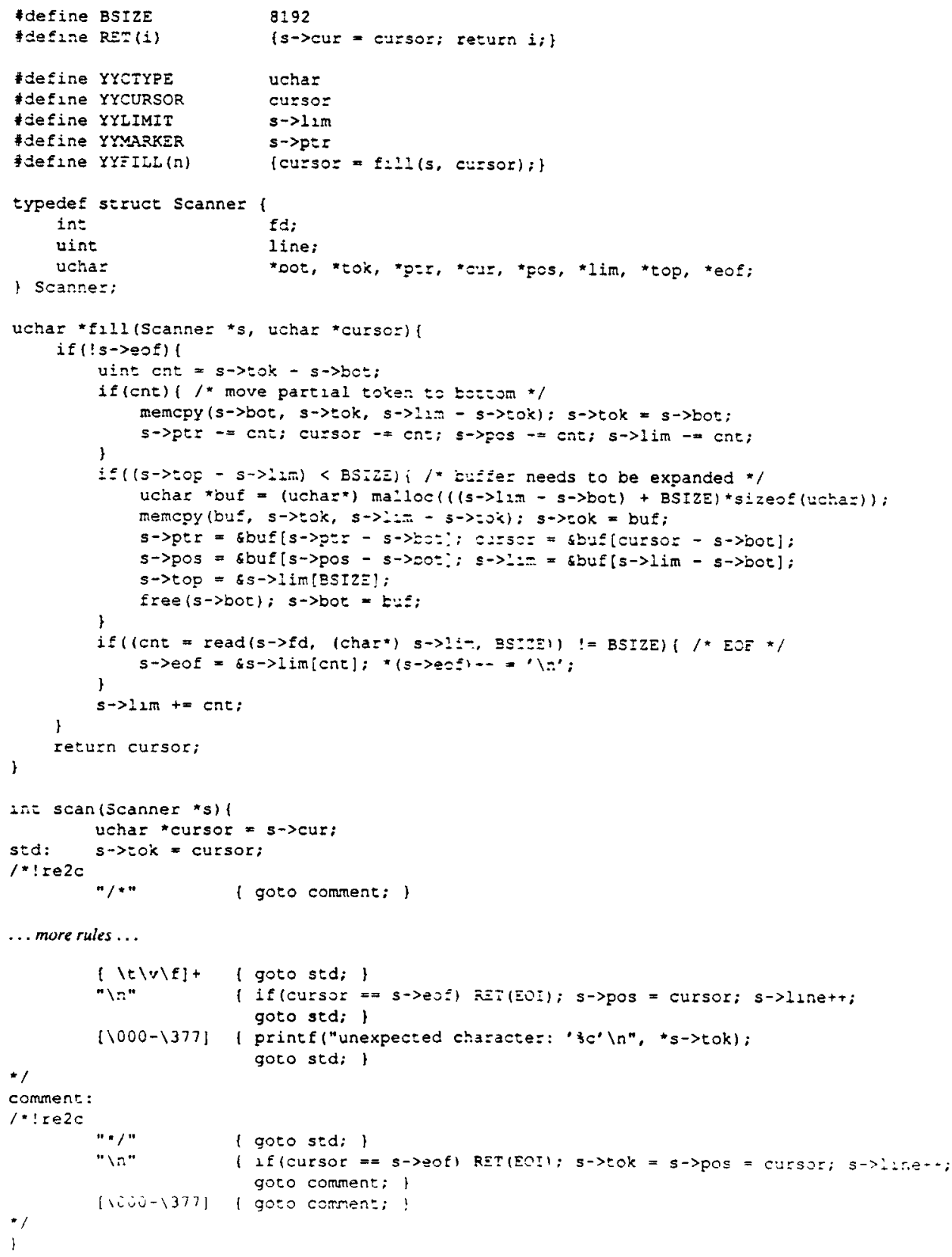

Figure 13

ACM Letters on Programming Languages and Systems, Vol. 2, Nos. 1-4, March-December 1993. 


\section{ACKNOWLEDGMENTS}

The authors thank the referees for their many valuable comments and suggestions.

\section{REFERENCES}

Aho, A. V., Sethi, R., And Ullman, J. D. 1988. Compilers: Principles, Techniques, and Tools. Addison-Wesley, Reading, Mass.

Bernstein, R. L. 1985. Producing good code for the case statement. Softw. Pract. Exper. 15, 10 (Oct.), 1021-1024.

DeRemer, F., AND Pennello, T. 1982. Efficient computation of LALR(1) look-ahead sets. ACM Trans. Program. Lang. Syst. 4, 4 (Oct.), 615-649.

Ellis, M., AND Stroustrup, B. 1990. The Annotated C++ Reference Manual. Addison-Wesley, Reading, Mass.

FRASER, C. W., AND HANSON, D. R. 1991. A retargetable compiler for ANSI C. SIGPLAN Not. (ACM) 26,10 (Oct.), 29-43.

GaReY, M. R., AND Johnson, D. S. 1991. Computers and Intractability: A Guide to the Theory of NP-Completeness. Freeman, San Francisco, Calif.

GRAY, R. W. 1988. $\gamma$-GLA-A generator for lexical analyzers that programmers can use. In USENIX Conference Proceedings (June). USENIX Association, Berkeley, Calif., 147-160.

Gray, R. W., Heuring, V. P., Levi, S. P., Sloane, A. M., and Watte, W. M. 1992. Eli: A complete, flexible compiler construction system. Commun. ACM 35, 2 (Feb.), 121-131.

Grosch, J. 1989. Efficient generation of lexical analysers. Softw. Pract. Exper. 19, 11, 1089-1103.

HaRRISON, M. A. 1978. Introduction to Formal Language Theory. Addison-Wesley, Reading, Mass.

Hennessy, J. L., And Mendelsohn, N. 1982. Compilation of the Pascal case statement. Softw. Pract. Exper. 12, 9 (Sept.), 879-882.

Horspool, R. N., AND WHITney, M. 1990. Even faster LR parsing. Softw. Pract. Exper. 20,6, $515-535$.

JACOBSON, V. 1987. Tuning UNIX Lex or it's NOT true what they say about Lex. In USENIX Conference Proceedings (Washington, D.C.). USENIX Association, Berkeley, Calif., 163-164. (Abstract only.)

Kernighan, B. W., AND Ritchie, D. M. 1988. The C Programming Language. 2nd ed. PrenticeHall, Englewood Cliffs, N.J.

LESK, M. E. 1975. LEX-A lexical analyzer generator. Comput. Sci. Tech. Rep. 39, Bell Telephone Laboratories, Murray Hill, N.J.

Paxson, V. 1988. flex-Man pages. In flex-2.3.7.tar.Z. (Available for anonymous ftp from ftp.uu.net in /packages / gnu.)

PENNELlo, T. J. 1986. Very fast LR parsing. In Proceedings of the ACM SIGPLAN86 Symposium on Compiler Construction (July). ACM, New York. SIGPLAN Not. 21, 7 (July).

SALE, A. 1981. The implementation of case statements in Pascal. Softw. Pract. Exper. 11, 9 (Sept.), 929-942.

Received February 1993; revised October 1993; accepted November 1993 